\title{
ANÁLISIS
}

\section{PRESENTE Y FUTURO DE LOS SISTEMAS RECOMENDADORES EN LA WEB 2.0}

\author{
Félix Pascual-Miguel, Julián Chaparro-Peláez y Antonio Fumero-Reverón
}

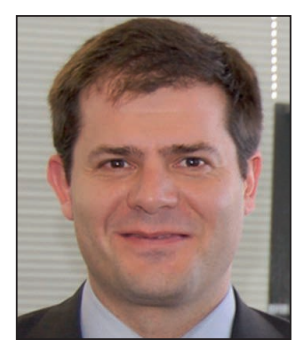

Felix Pascual-Miguel es profesor de organización de empresas de la ETSI de Telecomunicación de la Universidad Politécnica de Madrid (UPM). Doctor ingeniero de telecomunicación y MBA por la UPM. Imparte asignaturas de grado y posgrado en las áreas de dirección de empresas y sistemas de información empresariales. Ha dirigido y participado en diferentes proyectos de investigación nacional e internacional y es director y secretario académico de tres programas de posgrado de la UPM.

Universidad Politécnica de Madrid ETSI de Telecomunicación, desp. A-126 Av. Complutense, 30. 28040 Madrid felixjose.pascual@upm.es

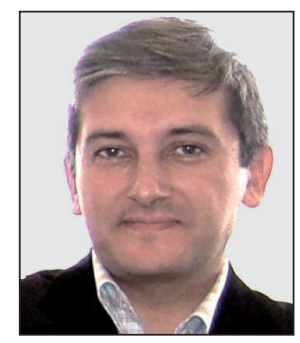

Julián Chaparro-Peláez es profesor titular de organización de empresas de la ETSI de Telecomunicación de la Universidad Politécnica de Madrid (UPM). Doctor ingeniero de telecomunicación, ha participado y dirigido numerosos proyectos de I+D con administraciones públicas y empresas en el ámbito de las tecnologías de la información y las comunicaciones y la organización de empresas. Ha dirigido tesis doctorales sobre sistemas de información y dirección de empresas, y es el coordinador del programa de doctorado de sistemas de información en la empresa de la UPM.

Universidad Politécnica de Madrid ETSI de Telecomunicación, desp. A-126 Av. Complutense, 30. 28040 Madrid julian.chaparro@upm.es

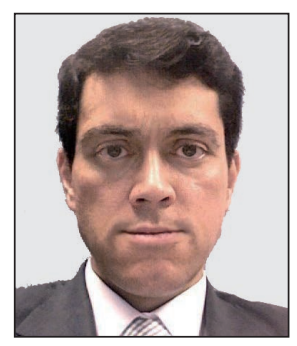

Antonio Fumero-Reverón es ingeniero de telecomunicación y MBA por la Universidad Politécnica de Madrid (UPM). Especialista en gestión de la tecnología e innovación, comenzó su carrera profesional en la Unidad de Desarrollo Tecnológico e Innovación del BBVA. Es investigador en la UPM, colaborando con la Cátedra Telefónica para Internet de Nueva Generación y el Grupo de Tecnologías de la Información para la Gestión Empresarial. Es socio fundador de Win-Win Consultores, y participa en otras iniciativas empresariales dentro del mismo ámbito, como TalentBrokers.

Universidad Politécnica de Madrid ETSI de Telecomunicación, Desp. A-126 Av. Complutense, 30. 28040 Madrid amfumero@gmail.com

\section{Resumen}

En una Web dominada por los medios sociales para la información, la relación y la comunicación, la dinámica que se establece entre contenidos, personas y tecnología cambia radicalmente. Ante la relevancia que cobra el contenido generado por usuarios en este escenario -esencialmente relacional-, la localización de las mejores fuentes de información requiere sistemas recomendadores que incorporen la naturaleza social de una Web que va más allá de la primigenia internet. Se revisan las aproximaciones actuales a los procesos de recomendación, poniéndolas en el contexto de las tendencias asociadas al fenómeno del social computing. Asimismo, se destacan algunas líneas de actuación en la redefinición del problema de la recomendación en un panorama dominado por las redes sociales y la generación de contenidos por los usuarios.

\section{Palabras clave}

Sistemas recomendadores, Sistemas de filtraje, Grafo social, Filtraje cooperativo, Redes sociales, Web 2.0, Fuentes de información, Contenidos, Web semántica.

\section{Title: Recommender systems as sources for information in web $\mathbf{2 . 0}$}




\begin{abstract}
In a Web dominated by social media for information, relationships and communication, the established dynamics between content, people and technology change radically. Given the relevance of the user-generated content in such a scenario and its essentially relational nature, successfully locating the best sources of information requires the development of recommender systems that incorporate the social characteristic of scenarios built on a network that goes beyond the original Internet. The article provides a review of current approaches to the process of recommendation, placing them in the context of consolidated trends associated with the phenomenon of Social Computing, and highlights some lines of development in the area of redefining the problem of recommendation in a context dominated by social networks and user-generated content.
\end{abstract}

\title{
Keywords
}

Recommender systems, Filtering systems, Social networks, Web 2.0, Collaborative filtering, Contents, Information sources, Semantic web, Social graph.

Pascual-Miguel, Félix; Chaparro-Peláez, Julián; Fumero-Reverón, Antonio. "Presente y futuro de los sistemas recomendadores en la web 2.0". El profesional de la información, 2011, noviembre-diciembre, v. 20, n. 6, pp. 645-651.

http://dx.doi.org/10.3145/epi.2011.nov.07

\section{Introducción}

La concepción original de la Web como una red de personas ha terminado sustanciándose con el fenómeno de la web 2.0, que ha provocado la adición del apelativo social prácticamente a toda la actividad en la Red. Los términos comunidad o red social (Fumero-Reverón; García-Hervás, 2008) se aplican hoy a la totalidad de los ecosistemas online. Las redes sociales se han convertido más en modelos organizativos (a nivel social, empresarial, institucional, etc.) que en simples servicios online (Freire, 2008).

La conceptualización de este escenario responde a la definición de Nuevo entorno tecnosocial (NET) propuesta por Fernando Sáez-Vacas (2004). Se trata de un panorama complejo, que surge y se ve afectado directamente por el desarrollo de lo que el mismo autor llama la red universal digital: "un conjunto heterogéneo en plena evolución compuesto por múltiples y diferentes redes (...), cada día más digitales e interoperables", que van mucho más allá de lo que popularmente conocemos como internet.

En este entorno han surgido los sistemas de recomendación (también llamados motores, marcos o plataformas de recomendación) que son un tipo específico de filtrado de información que intenta recomendar artículos (películas, programas de TV, vídeos, música, libros, noticias, imágenes, páginas web, publicaciones científicas, trabajos de investigación, etc.) o elementos sociales (personas, eventos o grupos) que pueden ser de interés para un usuario.

Por lo general, en un sistema de recomendación se compara un perfil de usuario con algunas de las características del artículo, producto o servicio, y se trata de predecir la preferencia (rating) que tal usuario tendrá por tal artículo que todavía no había considerado. Estas características pueden ser las del contenido (enfoque basado en el contenido, content-based approach) o las del entorno social del usuario (método de filtrado colaborativo o collaborative filtering).

El problema teórico de la recomendación se ha reducido tradicionalmente a la clasificación (rating) de los artículos (items) que son objeto de la recomendación. Consiste en la búsqueda del mejor sistema de correspondencia entre esos artículos y los usuarios, en el sentido de su clasificación por parte de éstos y de las similitudes entre los propios usuarios. De lo que se trata es de modelar el comportamiento del usuario para recomendarle contenidos y servicios siguiendo un proceso consistente y repetible.

Nuestra tesis de partida es que hay evidencias en este NET que nos llevan a reformular ese planteamiento teórico. Los nativos digitales hacen un uso intensivo de los sitios de redes sociales, y se dedican fundamentalmente a la socialización con sus iguales: "los jóvenes son conscientes de que internet les posibilita compartir contenidos generados por ellos mismos, lo cual hacen con el objetivo de estrechar lazos con sus amigos" (Sánchez-Navarro; Aranda, 2011).

Debemos precisar que el término "nativos digitales" se toma aquí con un sentido absolutamente pragmático: no se utiliza una definición generacional basada en un marco de edad, sino en un contexto tecnocultural concreto, el NET, y directamente relacionada con un uso intensivo y cotidiano de las tecnologías de social computing, cuya caracterización más detallada se puede ver en Gértrudix-Barrio et al. (2010).

Se trata de un escenario en el que además los investigadores son cada vez más conscientes del papel de la web 2.0 (Arroyo-Vázquez, 2009), y especialmente del contenido generado por los propios usuarios, como fuentes de información.

\section{Un sistema recomendador debe elegir qué objeto mostrar al usuario, y decidir cuándo y cómo mostrar las recomenda- ciones}

Hay que pensar por tanto en el problema de la recomendación desde la óptica de las redes sociales. Es decir, se trata de centrarnos más en la información que se extrae de las conexiones sociales de los usuarios y de las redes que ellos 
establecen, que en la información disponible en las propias piezas de contenido. Ello concede aún más relevancia si cabe a la percepción que de las herramientas y tecnologías propias de la web social tienen los usuarios en general y los investigadores en particular (Research information network, 2010), así como en la construcción de su propia identidad online (Reich, 2011).

¿Qué pasa si lo que se quiere recomendar es otra persona y no un artículo, una pieza de contenido o un servicio? ¿Se pueden mejorar los métodos de filtraje cooperativo existentes introduciendo ese componente social?

Se abordarán aquí las respuestas a estas cuestiones a partir de un repaso de los fundamentos y del estado del arte de los sistemas recomendadores desde un punto de vista lo suficientemente riguroso como para resultar de utilidad al lector interesado.

¿Qué pasa si lo que se quiere recomendar es otra persona y no un artículo, una pieza de contenido o un servicio?

\section{Sistemas de recomendación. Conceptos básicos}

Como se dijo, la clasificación tradicional de sistemas de recomendación distingue entre los métodos de filtraje basados en contenido y los cooperativos, dejando en medio un cada vez más amplio espectro de métodos híbridos que tratan de combinar creativamente elementos de ambos.

Los sistemas basados en contenido, como Google, que ha introducido en el mercado su enfoque conductual para los anuncios recomendados, utilizan métodos de análisis basados en texto, con grandes limitaciones en sus procedimientos para la extracción automática de metadatos. Además, tienen problemas con el usuario nuevo, es decir, con la situación que se produce cuando el sistema no acumula puntuaciones suficientes para emitir recomendaciones sobre items relevantes para el recién llegado.

http://www.google.com

Los sistemas de filtraje cooperativo, como Amazon, se basan en la extracción de información de los "iguales" (pares, aliados, amigos) de los usuarios objeto de la recomendación, generalmente a partir de las similitudes entre sus perfiles. Siguen teniendo problemas con la situación del recién llegado y con la inevitable escasez de usuarios en la fase de construcción de la comunidad, cuando todavía no se ha alcanzado la masa crítica necesaria.

http://www.amazon.com

Uno de los campos donde más ejemplos podemos encontrar es el de la música: Music genome project de Pandora, se basa en una caracterización detallada por expertos; Lala, que cerró en mayo de 2010, se apoyaba sólo en la comunicación entre usuarios; Mufin, sigue un modelo basado en contenido a partir de un algoritmo propio; y eMusic, ejemplo de enfoque híbrido, combina las recomendaciones directas entre usuarios con un algoritmo técnico e incluso con la participación de un grupo de "editores" que velan por la calidad de ciertas recomendaciones.
http://www.taringa.net/posts/links/18802/Pandora_Mu sic-Genome-Project.htm/

http://www.mufin.com/us/

http://www.emusic.com/

Una recomendación adecuada debe tener en cuenta no sólo factores de rendimiento, sino también elementos de contexto físico y social en la Red

Un sistema recomendador debería al menos: 1) elegir qué objeto se le debería mostrar al usuario; y 2) decidir cuándo y cómo se le deberían mostrar las recomendaciones. Para realizar una recomendación adecuada (Hernández-delOlmo; Gaudioso, 2008), debemos tener en cuenta no sólo factores de rendimiento (de los elementos componentes y del propio proceso de recomendación en su conjunto), sino también -y cada vez más- elementos de contexto: contexto físico (pensemos en la ubicuidad de los dispositivos móviles y los servicios asociados a los mismos) y social (relacionado con la información relevante de conducta y contacto que se puede extraer en las comunidades online).

\section{Hacia los sistemas de recomendación 2.0}

Entre los avances que aparecen en la bibliografía de los últimos años se incluye una mejor comprensión de los usuarios y de los artículos (items) a recomendar -que se pueden confundir en un escenario "social"- y la incorporación de la información contextual (Adomavicius; Tuzhilin, 2008) en el proceso de recomendación, el soporte de la clasificación multi-criterio o la introducción de métodos de recomendación más flexibles y menos intrusivos.

Es fácil ver la importancia del contexto en un sistema de recomendación al uso con algunos ejemplos:

- En un sistema de recomendación de películas hay que tener en cuenta: dónde se ve el filme o cómo se ve (cine, home cinema de casa de los padres, reproductor de DVD de la habitación...); con quién (padres, novia, amigos...); o cuándo (pre-estreno, sesión matinal...). Por ejemplo, un sistema recomendador complejo propone una cervecería en función de la localización geográfica y/o cercanía social de las personas con las que voy (información que podría obtener de los dispositivos móviles).

- La recomendación de las personas que se deberían conocer en una red social profesional.

- Los blogs que debería tener agregados un usuario en su lector de fuentes en función de las personas de su red con gustos similares y su historial de consumo de medios.

En lo que se refiere al conocimiento del usuario y de los items (artículos, piezas de contenido, servicios, etc.) es importante tener unos perfiles lo más completos posible y que se puedan introducir en los procesos de recomendación.

Otro de los elementos clave en la evolución de los actuales sistemas recomendadores es la clasificación multi-criterio (un sistema para la compra de casas debe tener en cuenta características como las calidades, precio, dimensiones, 
combinaciones de mobiliario, opciones de financiación, etc.). Se trata de un ámbito en el que se han utilizado métodos de toma de decisiones propios de la investigación de operaciones (Lee; Teng, 2007) para la mejora de los procesos de recomendación (Manouselis; Costopoulou, 2007).

\section{La intrusividad de los procesos de reco- mendación es otra de las grandes pre- ocupaciones}

La intrusividad de los procesos de recomendación es otra de las grandes preocupaciones en este área. Se suele buscar el equilibrio entre las clasificaciones y puntuaciones obtenidas automáticamente a partir de la interacción del usuario con los items y las que se obtienen a partir de la realimentación explícita de los propios usuarios. Una de las características que se espera de un escenario del tipo red social o comunidad es un mayor volumen de interacciones entre los usuarios, más ricas y con una mayor trazabilidad, lo cual debería ayudar a reducir el nivel de intrusividad en el proceso de recomendación.

Otra de las características que se demanda es una mayor flexibilidad. Que el usuario final pueda personalizar sus recomendaciones de acuerdo con sus necesidades en tiempo real. Desde el punto de vista técnico han aparecido algunas soluciones basadas en $R Q L$ (recommendation query language), de la misma forma que han aparecido algunas aproximaciones basadas en OLAP (online analytical processing) para la composición de recomendaciones agregadas (Jerbi et al., 2009).

La efectividad de las recomendaciones sigue siendo un problema. Las métricas disponibles parecen estar hasta cierto punto un tanto desfasadas: la cobertura y el grado de acierto no son suficientes para medir la bondad de los procesos de recomendación social que parece exigir el nuevo escenario que se da con la web 2.0. Algunas investigaciones recientes (Zheng; Wilkinson; Provost, 2008) dan a entender que en determinadas circunstancias el filtrado cooperativo basado en redes sociales puede arrojar mejores resultados que los métodos tradicionales de filtrado cooperativo (collaborative filtering, CF).

Esas circunstancias, aun no estando definidas con gran rigurosidad, hacen pensar en un escenario en el que:

a) los items que debemos recomendar son personas que forman parte de la misma red social

b)el método de clasificación para efectuar la recomendación podría ser multi-criterio, incluyendo conectividad, similitud en términos de perfil y/o comportamiento (de compra, por ejemplo, en una comunidad como eBay) y reputación de las personas objeto de recomendación.

El móvil "inteligente" es, sin lugar a dudas, el elemento estelar (Rodríguez-Sánchez; Sáez-Vacas; García-Hervás, 2010) en este panorama identificado conceptualmente con el NET definido por Sáez-Vacas (2004). En lo que se refiere por tanto al problema de la recomendación, la cuestión que este hecho plantea es ¿cómo podemos recomendar contenidos, personas o servicios en movilidad? La respuesta no es trivial.
En este escenario el contexto cobra más importancia a la hora de realizar recomendaciones eficaces. ¿Qué pasa si incluimos las distancias entre los nodos en el proceso de recomendación? No hace mucho se empezaba a hacer algo parecido con el lanzamiento de buscadores sociales que incorporaban ciertos componentes de filtraje cooperativo y elementos de red social al proceso de búsqueda, que tradicionalmente dependía únicamente de la bondad del algoritmo implementado por nuestro motor de búsqueda favorito.

Algunos ejemplos son: HayStack que proponía compartir las experiencias de búsqueda de sus usuarios, o Porqpine que iba un paso más allá, funcionando como una comunidad con un interés común en mejorar esas experiencias de búsqueda.

http://en.wikipedia.org/wiki/Haystack_\%28MIT_pro ject\%29

http://www.w3.org/2005/04/sw/s/BioDash/Demo/ What\%20is\%20Haystack.html

Esas ideas -basadas en la introducción de nuestro contexto "ambiental" implícito en el proceso de recomendación-son las que han impulsado el desarrollo del concepto de ambient recommendation (González et al., 2006).

\section{En un escenario 2.0 hay que centrarse más en la información de las conexiones sociales de los usuarios que en la infor- mación de los artículos que estamos re- comendando}

El desafío sigue estando en explicitar las recomendaciones que realizan los usuarios implícitamente en la multitud de sitios de redes sociales que pueblan la Red, sin que tengan que utilizar conscientemente un sistema recomendador. Puede ocurrir que un usuario que necesita una recomendación no siempre sea capaz de explicitarla en los términos en que un sistema recomendador al uso necesitaría. La realidad de las redes sociales convierten todo el ecosistema de sitios que ofrecen esos servicios en la Web (sitios de redes sociales, SRS) en un gigantesco sistema de recomendación basado en un proceso virtual e implícito de transmisión de "boca a oreja" (electronic word-of-mouth, eWoM) que busca realimentar los procesos explícitos de recomendación con la sensibilidad de los propios usuarios a las recomendaciones implícitas, puesto que se ha constatado que "los usuarios sensibles a las recomendaciones implícitas parecen considerar los SRS como un canal de eWoM apropiado para las recomendaciones explícitas" (Ebermann; Stanoevska-Slabeva; Wozniak, 2011).

Si nos creemos que, efectivamente, estamos frente a un escenario nuevo, más social, tampoco es difícil ver que necesitamos una forma más o menos coherente y consistente para representarlo. Uno de los conceptos que más popularidad ha ganado entre la comunidad investigadora es el "grafo social", entendido como un mapa que incluye información de las personas (nodos) que forman parte de una red y de cómo están relacionadas unas con otras (conexiones). Era 
el propio Tim Berners-Lee quien bromeaba proponiendo la necesidad de considerar la Red que hoy conocemos más como un GGG (global giant graph) que como una WWW, destacando precisamente su naturaleza reticular y social como elementos relevantes para su estudio.

Si bien esos elementos pueden actuar como una especie de infraestructura básica, está claro que necesitaremos otros elementos más operativos para soportar un proceso eficaz de identificación en la Red que vaya más allá de iniciativas como OpenID, un esquema blando de identidad distribuido u OAuth, pensado para gestionar el acceso a recursos, en su concepción más amplia. Iniciativas como Open social, también del gigante Google, o Data portability que inciden en el problema de los datos habitualmente incluidos en los tan populares perfiles de redes sociales, tampoco han conocido aún una gran proyección, siendo elementos que podrían jugar un papel importante en el escenario de las recomendaciones sociales.

\section{Mirando al futuro}

Estamos habituados a hablar de la relación que se establece entre los usuarios y los items o artículos a través de la clasificación o compra de estos últimos por parte de los primeros. Pero: ¿Qué pasa en un sitio genérico de redes sociales donde esos items son otros usuarios? ¿Qué pasa si consideramos que la relación de compra se hace efectiva cuando se establece una conexión entre dos usuarios? ¿Podemos recomendar usuarios a otras personas dentro de una red social?

El desafío está en explicitar las recomendaciones que realizan los usuarios implícitamente en multitud de redes sociales, sin utilizar conscientemente un sistema recomendador

El trabajo de Zheng, Wilkinson y Provost (2008) considera este tipo de evidencias y supuestos, partiendo de una diferencia sustancial básica entre el filtrado cooperativo tradicional y el basado en redes sociales, establecida en términos de complejidad computacional, algorítmica y el problema clásico del "arranque frío" (cold start) que afronta el sistema recomendador cuando no se ha alcanzado una masa crítica de usuarios. La experimentación que llevan a cabo se apoya en un sitio concreto de redes sociales donde se comparten ideas y posiciones políticas, de tal manera que ofrece cierta versatilidad para definir la similitud o afinidad entre usuarios para establecer enlaces entre ellos -buscando contrarios, aliados y amigos.

Los resultados obtenidos por Rong Zheng (2008) avalan la consistencia de una línea de investigación clásica ya (Kautz; Selman; Shah, 1997) que parte de la idea recíproca de la planteada anteriormente, es decir extraer enlaces "sociales" a partir de la historia compartida de clasificación o compra de varios usuarios. El estudio de la propagación de las recomendaciones a lo largo y ancho de una red social o de cómo se transmite la "confianza" a través de las conexio- nes explícitas e implícitas de una comunidad han dado lugar ya al replanteamiento de los sistemas de recomendación desde el punto de vista de cómo se modela al usuario para conectarlo con otros usuarios, no para recomendarle items o artículos que comprar (Perugini; Gonçalves; Fox, 2004).

A la hora de pensar en una implementación práctica para el usuario habitual de los sitios de redes sociales (social networking sites, SNS), lo primero que uno se encuentra es la necesidad de construir, técnicamente, la red social sobre la que trabajar. Podríamos pensar en una implementación para Twitter (red a la que se atribuyen más de 200 millones de usuarios, y donde el proxy social utilizado, el "seguimiento" de otros usuarios, "follow", resulta más ilustrativo si cabe) o Facebook (el SNS más popular que las estadísticas oficiales sitúan con más de 500 millones de usuarios activos, que se convierten en más de 750 millones según otras fuentes).

¿Podemos extraer información suficiente para elaborar un grafo social con ciertas garantías? Joseph Bonneau et al. (2009) afirmaban que "con ocho (amigos) basta". En una experiencia orientada a destapar ciertas vulnerabilidades de los elementos de privacidad en las redes sociales al uso, sacando partido de la lista aleatoria de ocho contactos que se podía obtener con los resultados de la búsqueda de un perfil de usuario cualquiera en Facebook, llegaban a construir una aproximación del grafo social cuya bondad constataban mediante un conjunto de medidas estándar (centralidad, caminos más cortos, conjuntos dominantes, etc.) consiguiendo un grado de aproximación realmente preocupante con respecto a las medidas exactas que se obtenían a partir del grafo completo.

A partir de otra propuesta práctica, como es el caso de recomendar piezas de información en formato blog para su consumo (lectura, visionado, reproducción), ya sea directamente o a través de un agregador lector de fuentes en formato estándar para sindicación (Google Reader) podemos prever la complejidad que supone la definición de una métrica multi-criterio para la clasificación de ese tipo de contenido: la propuesta preliminar que recogía Mary Hodder (2005) para el diseño de una métrica del formato blog puede ser un buen ejemplo para el lector. Hay que tener en cuenta, además, que este tipo de plataformas y formatos no se orientan hoy tanto al contenido como a la conectividad en red de productores y consumidores de contenido generado por otros usuarios.

\section{Conclusiones}

Visto ese escenario y las tendencias de los sistemas de recomendación, el desarrollo de la línea de filtraje cooperativo basado en redes sociales como una de las más prometedoras en la evolución de los sistemas recomendadores tal y como los conocemos, nos lleva a plantearnos algunas cuestiones, al hilo también de algunos de los trabajos que se han revisado en el artículo.

El hecho de que los procesos de filtraje cooperativo que se basan en la extracción de similitudes a partir del grafo social obtengan, en media, resultados sólo ligeramente peores que un proceso tradicional nos hace pensar que un escenario en el que se generaliza el uso de los servicios online 
para la gestión de redes sociales puede ser el adecuado para introducir cierto nivel de automatización en los servicios de recomendación.

La posibilidad de generar funciones (scoring, rating, reputación, influencia, etc.) sobre un grafo social parcial con la seguridad (matemática y computacional) de que vamos a obtener resultados similares (con un grado de confianza que se puede determinar) puede justificar el uso de información "pública" obtenida de los sitios de redes sociales (SRS) genéricos (Twitter, Facebook o LinkedIn) para ensayar algunas de esas funciones y su efecto en el rendimiento de los procesos de filtraje cooperativo basados en redes sociales. No es difícil pensar en la búsqueda de usuarios similares a partir de la información del grafo social y algún tipo de función de reputación que podamos definir sobre las vistas parciales que podamos obtener sobre los diferentes SRS a partir de sus interfaces de aplicación (API, Application programming interface).

Si consideramos, por ejemplo, en una red de blogs en que los items son contenidos digitales y la relación de compra supone el consumo de los mismos, que a los efectos de presentar una propuesta práctica se pueda simplificar como la lectura de entradas en un blog, podemos pensar en recomendar "cosas que leer" en un agregador para fuentes de información publicadas en formato estándar para la sindicación de contenidos (RSS, Atom).

En cuanto al modelo de implementación, hay que tener en cuenta al menos:

- diseño de un mecanismo para la extracción del grafo social a partir de los metadatos que ofrecen los perfiles de los sitios de redes sociales:

- definición de una clasificación multi-criterio que se adecue al ítem recomendado;

- métricas de rendimiento específicas para los procesos de filtrado cooperativo basados en redes sociales.

\section{Referencias}

Adomavicius, Gedimias; Tuzhilin, Alexander. "Toward the next generation of recommender systems: a survey of the state of the art and possible extensions". IEEE transactions on knowledge and data engineering, 2005, junio, v. 17, n. 6, pp. 734-749.

http://ids.csom.umn.edu/faculty/gedas/papers/recom mender-systems-survey-2005.pdf

http://dx.doi.org/10.1109/TKDE.2005.99

Adomavicius, Gedimias; Tuzhilin, Alexander. "Contextaware recommender systems". En: ACM conf on recommender systems. Lausanne, Suiza, 2008, pp. 335-336. ISBN 9781-60558-093-7

http://dx.doi.org/10.1145/1454008.1454068

Adomavicius, Gediminas; YoungOk, Kwon. "New recommendation techniques for multi-criteria rating systems". IEEE intelligent systems, 2007, v. 22, n. 3, pp. 48-55.

http://www.misrc.umn.edu/working papers/fullpa pers/2006/0608_120506.pdf

http://dx.doi.org/10.1109/MIS.2007.58
Agrahri, Arun-Kumar; Manickam, Divya-Anand-Thattandi; Riedl, John. "Can people collaborate to improve the relevance of search results?" En: ACM conf on recommender systems. Lausanne, Suiza, 2008, pp. 283-286. ISBN 978-160558-093-7

http://www.grouplens.org/system/files/p283-agrahri.pdf http://dx.doi.org/10.1145/1454008.1454052

Arroyo-Vázquez, Natalia. "Fuentes de información en la web social en biblioteconomía y documentación". En: XI Jornadas españolas de documentación. Zaragoza: 20-22 mayo, 2009, pp. 87-98.

http://hdl.handle.net/10760/13162

Bonneau, Joseph; Anderson, Jonathan; Anderson, Ross; Stajano, Frank. "Eight friends are enough: social graph aproximation via public listings". En: Procs of the Second ACM EuroSys workshop on social network systems. Nuremberg: ACM, 2009, pp. 13-18. ISBN 9781605584638 http://dx.doi.org/10.1145/1578002.1578005

Ebermann, Jana; Stanoevska-Slabeva, Katarina; Wozniak, Thomas. "Influential factors of recommendation behaviour in social network sites - an empirical analysis". En: ECIS 2011 Procs, paper n. 259

http://aisel.aisnet.org/ecis2011/259/

Freire, Juan. "Redes sociales: ¿modelos organizativos o servicios digitales?". El profesional de la información, 2008, noviembre-diciembre, v. 17, n. 6, pp. 585-588. http://dx.doi.org/10.3145/epi.2008.nov.01

Fumero-Reverón, Antonio; García-Hervás, José-Miguel. "Redes sociales". Telos, 2008, julio-septiembre, n. 76, pp. 49-131.

http://sociedadinformacion.fundacion.telefonica.com/telos/ cuaderno.asp@rev=76.htm

Gértrudix-Barrio, Felipe; Durán-Medina, José-Francisco; Gamonal-Arroyo, Roberto; Gálvez-de-la-Cuesta, Maríadel-Carmen; García-García, Francisco. "Una taxonomía del término 'nativo digital': nuevas formas de relación y de comunicación”. Sala de prensa, 2010, mayo, v. 6, n. 127. http://www.saladeprensa.org/art998.htm

González, Gustavo; De-la-Rosa; Josep-Lluís; Dugdale, Julie; Pavard, Bernard; El Jed, Mehdi; Pallamin, Nico; Angulo, Cecilio; Klann, Markus. "Towards ambient recommender systems: results of new cross-disciplinary trends". En: Felfernig, Alexander; Zanker, Markus (eds.) Procs of 17th European conf on artificial intelligence (ECA/2006), pp. 128-133. Workshop on recommender systems. Riva del Garda, Italia, 2006, 28-26 agosto.

http://magma.imag.fr/publications/papers/Gonzalez-etal06.pdf

Hernández-del-Olmo, Félix; Gaudioso, Elena. "Evaluation of recommender systems: a new approach". Journal expert systems with applications, 2008, octubre, v. 35, n. 3, pp. 790-804.

http://dx.doi.org/10.1016/j.eswa.2007.07.047

Hodder, Mary. Link love lost or how social gestures within topic groups are more interesting than link counts. http://napsterization.org/stories/archives/000513.html 
Lee, Hsin-Hsien; Teng, Wei-Guang. "Incorporating multi-criteria ratings in recommendation systems". IEEE intl conf on information reuse and integration, 2007, pp. 273-278. http://dx.doi.org/10.1109/IRI.2007.4296633

Jerbi, Houssem; Ravat, Franck; Teste, Olivier; Zurfluh, Guilles. "Applying recommendation technology in OLAP systems". En: Filipe, Joaquim; Cordeiro, José. Enterprise information systems. Berlin Heilderberg: Springer-Verlag, 2009, v. 24, n. 1, pp. 220-233. ISBN 978-3-642-01346-1 http://www.springerlink.com/content/k674v90734/73m71/ http://dx.doi.org/10.1007/978-3-642-01347-8_19

Juárez-Urquijo, Fernando. "Evolución de la web social en 2010. Informe de situación“. Anuario ThinkEPI, 2011, v. 5, pp. $146-154$

Kautz, Henry; Selman, Bart; Shah, Mehul. "Referral web: combining social networks and collaborative filtering". Communications of the ACM, 1997, v. 40, n. 3, pp. 63-65.

http://www.cs.cornell.edu/selman/papers/pdf/97.cacm. refweb.pdf

http://dx.doi.org/10.1145/245108.245123

Manouselis, Nikos; Costopoulou, Constantina. "Analysis and classification of multi-criteria recommender systems". World wide web journal, 2007, v. 10, n. 4, pp. 415-441.

http://www.springerlink.com/content/v703763243148332 http://dx.doi.org/10.1007/s11280-007-0019-8

Perugini, Saverio; Gonçalves, Marcos-André; Fox, Edward. "Recommender system research: a connection-centric survey". Journal of intelligent information systems, 2004, v. 23, n. 2, pp. 107-143.

http://www.springerlink.com/content/r322040289831534 http://dx.doi.org/10.1023/B:JIIS.0000039532.05533.99

Pujol, Josep M.; Rodríguez, Pablo. "Towards distributed social search engines". Telefónica Research. In: WWW2009, April 20-24, 2009, Madrid, Spain. http://www2009.eprints.org/242/1/porqpine-www2009developers.pdf

Quercia, Daniele; Hailes, Stephen; Capra, Licia. "MobiRate: making mobile raters stick to their word". Ubicomp, 2008. http://www.cs.ucl.ac.uk/staff/d.quercia/publications/quer cia08mobirate.pdf

Reich, Eugenie-Samuel. "Online reputations: best face forward". Nature, 2011, n. 473, pp. 138-139.

http://dx.doi.org/10.1038/473138a

Research information network. If you build it, they will come? How researchers perceive and use web 2.0.

http://www.rin.ac.uk/system/files/attachments/web_2.0_ screen.pdf

Rodríguez-Sánchez, Francisco; Sáez-Vacas, Fernando; García-Hervás, José-Miguel. El teléfono móvil, producto estelar de la red universal digital. Una tecnología compleja de impresionante y ubicuo impacto social. Madrid: Cátedra Orange - UPM, 2010.

http://jungla.dit.upm.es/ jsr/ElTelefonomovilproductoestel ardelaRUDv2.2.pdf

Sáez-Vacas, Fernando. Más allá de internet: la Red universal digital. Madrid: Editorial Centro de Estudios Ramón Areces, 2004. ISBN 8480046295.

Sánchez-Navarro, Jordi; Aranda, Daniel. "Internet como fuente de información para la vida cotidiana de los jóvenes españoles". El profesional de la información, 2011, v. 20, n. 1, pp. 32-37.

http://eprints.rclis.org/bitstream/10760/15332/1/032-037.pdf http://dx.doi.org/10.3145/epi.2011.ene.04

Zheng, Rong; Wilkinson, Dennis; Provost, Foster. Social network collaborative filtering. Working Paper CeDER-8-08, 2008.

http://www.hpl.hp.com/research/scl/papers/sncf/sncfwp.pdf

\section{IraLIS es el registro de autoridades de nombres de autor del repositorio E-LIS}

\section{Antes de subir documentos a E-LIS debes iralizarte siguiendo los "Criterios de firma".}

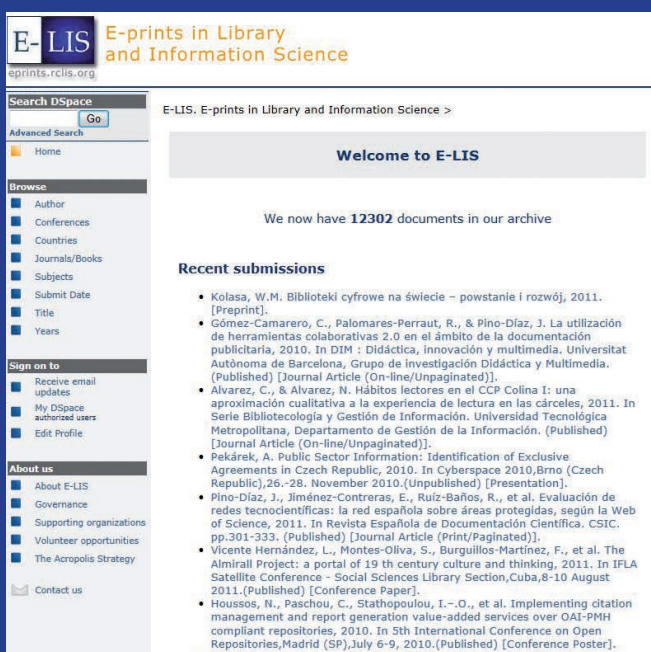

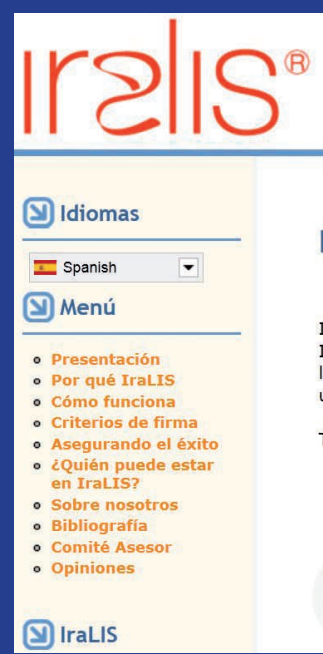

International Registry for Authors Links to Identify Scientists

Presentación

IraLis (International Registry of Authors-Links to Identify Scientists) es un sistema de estandarización de las firmas de los autores científicos. Su objetivo es crear un registro de autoridades.

Trabaja en 5 lineas:

1. Concienciar a los autores científicos sobre la importancia de la firma para ser citados correctamente, y para poder recuperar toda la bibliografía a lo largo de su carrera profesional.

2. Redactar criterios de firma normalizada para b) distinguirse de otros autores con nombres 